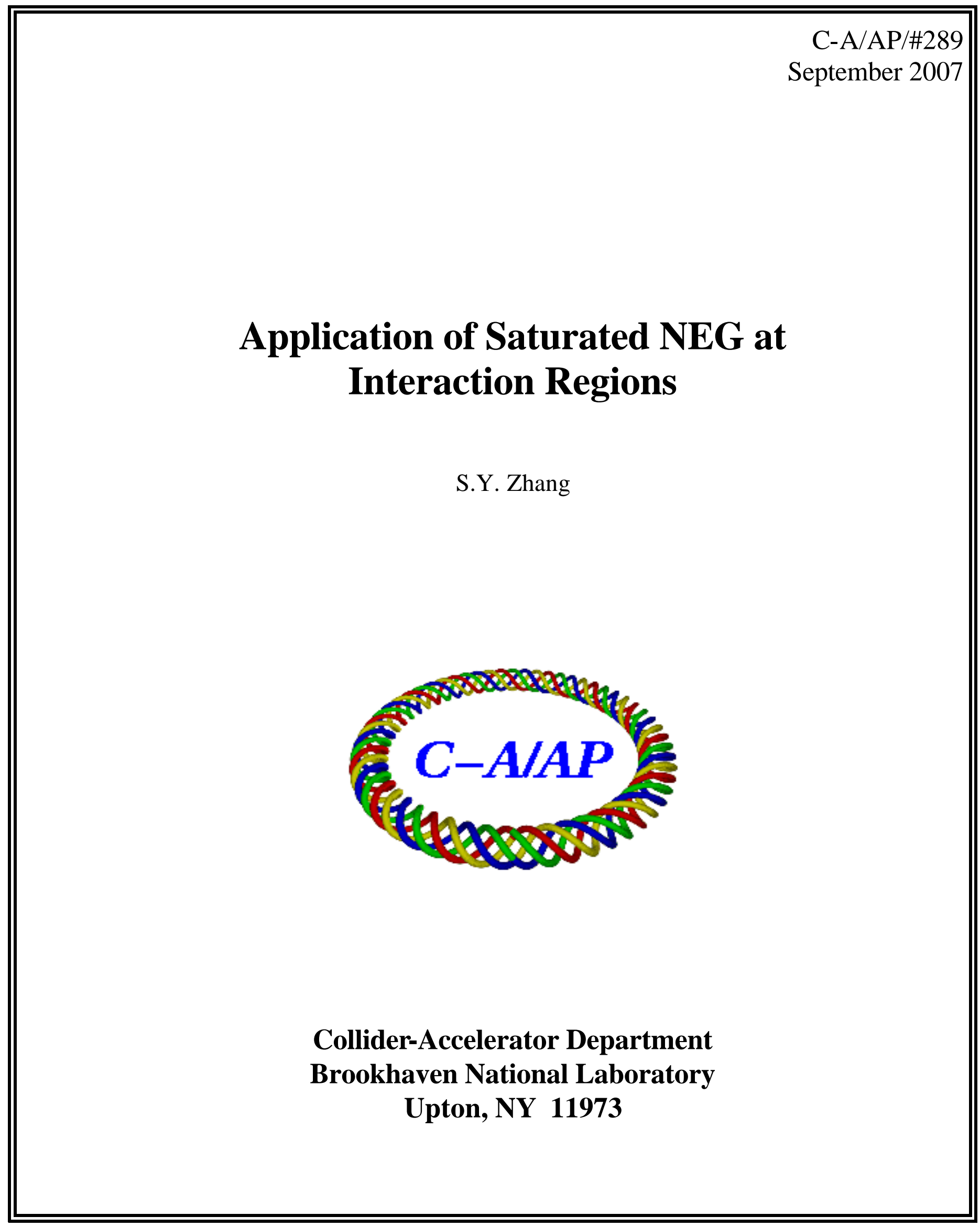




\title{
Application of Saturated NEG at Interaction Regions
}

\author{
September 21, 2007
}

\section{Abstract}

Activated non evaporable getter (NEG) coating has shown excellent surface properties, such as low secondary electron yield and low electron impact desorption rate, etc. Since usually the activation is difficult or sometimes not possible at interaction regions, the activated then saturated NEG coating could be used there, which does not require in-situ activation while still keeps many attractive surface properties. In this article, the experimental background problem at RHIC and the surface property of NEG coating are reviewed. The issues relevant to the application of saturated NEG at interaction regions are discussed.

\section{Introduction}

The non evaporable getter coating was developed primarily for the linear pumping [1], however, it was found that the activated NEG surface can also suppress the gas desorption induced by photon and electron bombardments [2]. Furthermore, the NEG surface has shown the reduced secondary electron yield (SEY) [3], and also the reduced desorption rate by ion impact [4]. The surface property of NEG coating (at least SEY) is probably only slightly changed after the saturation [3], which means that the low SEY is kept at the saturation.

The surface property of beam chamber at interaction regions is an important aspect in improving experimental background. Since the NEG activation 
at interaction regions is often limited in temperature or even not possible at all, the use of saturated NEG is of interest.

To this end, some questions need to be answered.

1. What surface property is relevant in the reduction of experimental background? These properties include SEY, electron and ion desorptions, and beam halo scrapping effects.

2. What do we know about these surface property for NEG coating and the saturated NEG coating?

3. How to improve the relevant surface properties in terms of coating and saturation methods and conditions? How to measure it?

4. Other questions such as the aging and lifetime of saturated NEG, and the installation and maintenance as well.

In this article, the RHIC experimental background problem in heavy ion and polarized proton operations is reviewed and several concerned surface properties of beam chamber at interaction region are discussed. This is followed by a review of NEG coating properties according to the bench test and machine studies performed at various laboratories. The issues that are not sufficiently understood are then discussed for further development.

\section{Experimental background}

\section{$3.1 \quad$ RHIC experience}

Luminosities of RHIC heavy ion operations are affected by the experimental background problem in past several years, including collisions of $\mathrm{Au}-\mathrm{Au}, \mathrm{d}-$ $\mathrm{Au}$, and $\mathrm{Cu}-\mathrm{Cu}$.

Since the bunch intensity has a quadratic relation to the luminosity, beams with highest possible bunch intensity are used as far as the beambeam and inter-beam scattering limits are not reached, which is the case for past and the current RHIC operations. The number of bunches then can be increased to raise luminosity. In $2003 \mathrm{~d}$-Au run, however, the bunch number had to be reduced from 110 to 55 [5]. In $2004 \mathrm{Au}-\mathrm{Au}$ run, most times 45 bunches was used [6]. In $2005 \mathrm{Cu}-\mathrm{Cu}$ run, only 37 to 40 bunches were used 
[7]. Beyond these limits, the experiments could not take data due to high background.

The RHIC polarized proton run currently is not limited by the background problem, but the problem is not irrelevant [8].

In the following, two cases are shown to illustrate the RHIC experimental background problem in heavy ion and proton operations.

In Figure 1, a typical case in Au-Au run 2004 is shown. With 45 bunches (average bunch spacing of $267 \mathrm{~ns}$ within the bunch train) and average bunch intensity of $1.07 \times 10^{9}$ fully stripped gold ions in Fill 4832, the pressure at the experiment PHOBOS beam pipes increased to above $2 \times 10^{-10}$ Torr from the static pressure of $3 \times 10^{-11}$ Torr. After a period of about 1 hour, the pressure suddenly dropped to below $10^{-10}$ Torr, perhaps due to the elimination of electron multipacting conditions. During this high pressure rise period, the ratio of the background (singles) and the ZDC (Zero Degree Calorimeter coincident rate) was higher than 20, PHOBOS could not take data. After this period, this ratio was reduced to below 15 , PHOBOS could start to take data, although the ideal condition is the ratio below 10 .

The machine operation condition had to be compromised with this background problem. For bunch number higher than 45, the PHOBOS would suffer much more, and for less bunches other experiments would take a larger reduction of the luminosity.

Fill 7621 in 2006 polarized proton run is used to illustrate the background problem for the experiment STAR. With 110 bunches (average bunch spacing of $108 \mathrm{~ns}$ ) and average bunch intensity $0.82 \times 10^{11}$ protons, the background at STAR was not a problem after collimations. STAR normally takes data with its central magnet on, which provides a uniform solenoid field of about $4.8 \mathrm{kGauss}$ at \pm 4 meters from the collision point. This strong solenoid field effectively suppresses the electron multipacting at STAR beam pipe, and hence the beam induced pressure rise is normally below $10^{-9}$ Torr. Note that the beam-gas cross section of proton is much smaller than the gold, therefore, this pressure rise is tolerable.

The STAR, however, is also interested in taking data with zero-field. In Figure 2, the pressure rise, the background and ZDC are shown for a case with STAR magnets off. The pressure rises from below $2 \times 10^{-11}$ Torr to above $5 \times 10^{-8}$ Torr. The peak background rate was higher than $1.7 \mathrm{MHz}$. The contaminated ZDC rate reached $250 \mathrm{kHz}$, which was not real coincidents. The trustful coincident rate at this intensity with usual beam emittance of 17 $\pi \mu m$ should be about $5 \mathrm{kHz}$. This is shown after the STAR magnets ramped 



Figure 1: Pressure rise and background of PHOBOS for $\mathrm{Au}-\mathrm{Au}$ run, Fill 4832. The background/ZDC ratio was larger than 20 when the pressure at the interaction region was higher than $2 \times 10^{-10}$ Torr. During this period (about 1 hour in this case) the PHOBOS could not take data. After the pressure reduced to less than $10^{-11}$ Torr, the background was reduced, and PHOBOS could start to take data. 
up and after some beam steering and collimations.

It is clear that if STAR needs to take zero-field data, the bunch intensity and/or bunch number need to be reduced drastically, which means significant luminosity reduction.

\subsection{Background reduction}

The experimental background comes from several sources [5].

1. Beam-beam. Background would rise when the bunches were cogged and the beam put in collision, due to single Coulomb dissociation. Since this background is somewhat inherent, one has to live with it.

2. Beam-chamber interaction. The resulted neutrons and other secondary particles from the beam-chamber interactions would cause background problem. For the interactions taken place at a distance from the interaction point, collimation and shielding could help. For the ones taken place close to the interaction point, collimation could help to reduce the halo, but better chamber surface is also relevant. The better surface here implies less secondary production of electrons, ions, and neutral particles. The incident type is the beam scraping, which are with very shallow incident angles.

3. Beam-gas. The background produced by beam-gas interaction depends on the density of the gas and the intensity of the beam. The latter, of course, is also associated with luminosity. To reduce the gas density is therefore the target of the mitigation. Usually the minimum static pressure at interaction regions is a matter of machine design. Therefore the target is the gas generated by the beam induced electron and ion impacts on the wall, which has multipacting effects. These are normal incidents with perpendicular impact angles. The surface of the chamber is crucial in reducing the effect of these impacts.

One may consider therefore the following aspects to reduce the background through the chamber surface improvement.

1. To reduce SEY, which might be the most important factor in the current RHIC experimental background reduction. It is the normal incident and the projectile and secondary product are both electrons. 

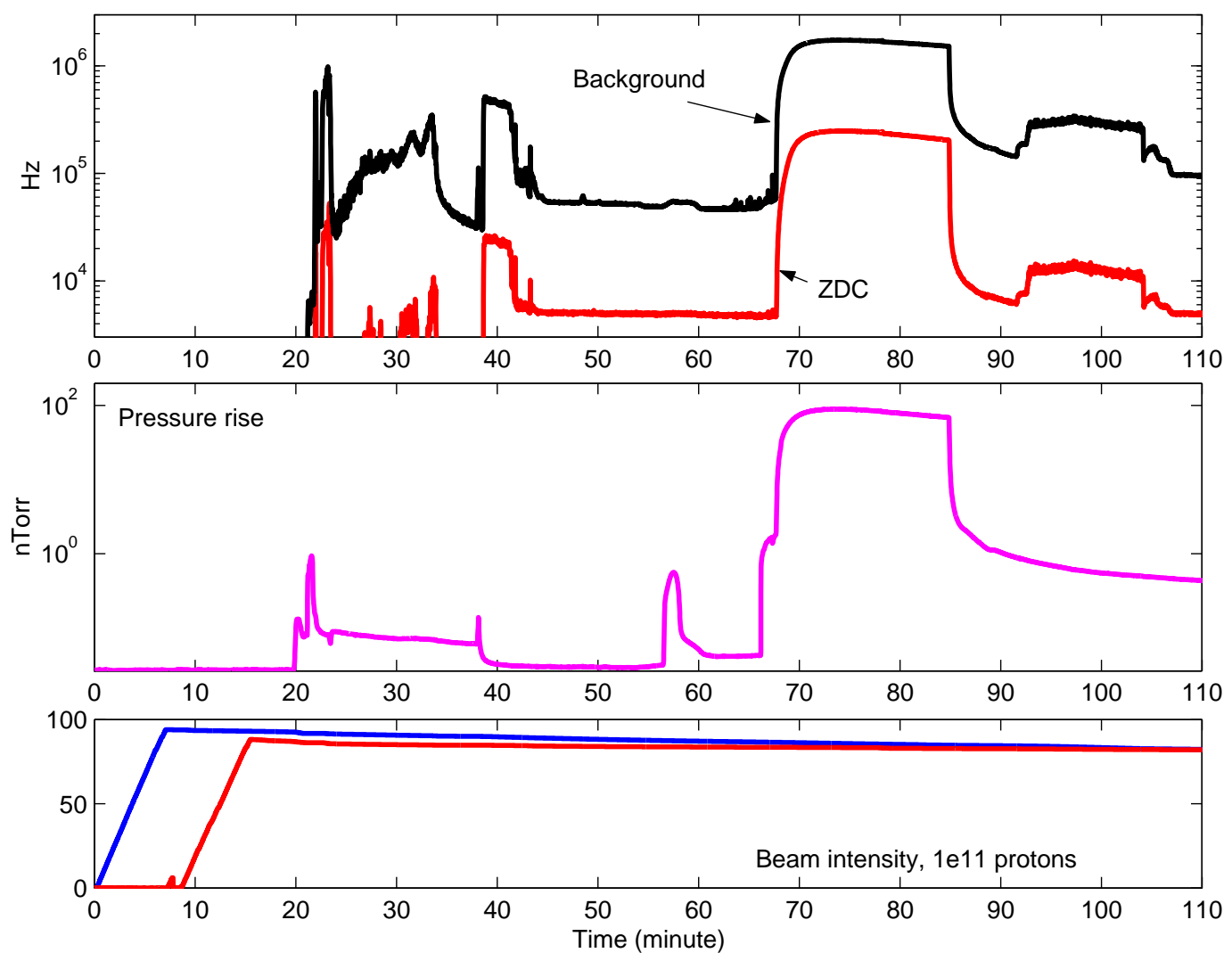

Figure 2: Beam intensity (bottom), STAR pressure rise (middle), STAR background and ZDC (upper) for Fill 7621 in proton run. The pressure rise from 20 to 40 minutes are due to the beam acceleration. The STAR magnets ramped down and up again during 55 to 90 minutes. The STAR background rises at the pressure higher than $10^{-9}$ Torr. Between 90 to 105 minutes, the background was not due to beam-gas, but due to beam steering. At the end (110 minutes), the ZDC was $4.7 \mathrm{kHz}$, which is about right. 
2. To reduce electron desorption rate, which might be the second most important aspect in RHIC problem. It is the normal incident and the secondary products are neutral particles and ions.

3. To reduce ion desorption rate. It is the normal incident and the secondary products are neutral particles and ions. The primary ions in the chamber come from beam-gas interaction, electron impact on the wall, and also from the beam-chamber interactions. These ions would be pushed to wall in a much slower rate than electrons, say in a few $\mu s$, and hitting the wall with the energy between a few $\mathrm{eVs}$ to a few hundreds $e V \mathrm{~s}$, depending on the ions, chambers, and beam intensities and emittances.

4. To reduce secondary effects of beam halo scraping on the wall. These are incidents with angles much smaller than $1 \mathrm{mrad}$, perhaps in $\mu \mathrm{rads}$. The secondary products include electrons, ions, and neutral particles.

\section{Surface property of NEG coating}

Some of the NEG coating surface property relevant to experimental background reduction have been studied, and understandings with different degrees have been reached. In this section, these results will be reviewed.

\subsection{SEY}

For TiZrV thin film coated on copper with $1 \mu \mathrm{m}$ thickness, after 2 hours of activation at $200^{\circ} \mathrm{C}$, the peak SEY has reduced from 2.0 to 1.1. Saturating the NEG coating with CO increased SEY by just 0.1 [3]. These results are confirmed at least in some degrees by the beam test at SPS and RHIC. At SPS, the usual electron multipacting disappeared at the activated and saturated NEG pipes [9]. At RHIC, the dynamic pressure rise, which is mainly caused by the electron cloud, is drastically reduced at the straight sections with NEG coating [10]. NEG coating at one section at RHIC was saturated by $N_{2}$, and the suppression of electron cloud is unchanged [11].

As comparison, SEY of steel surface could be reduced to 1.2 after electron dose of $1 \mathrm{mC} / \mathrm{mm}^{2}$ [12], which is a rather large dose and would require very long time in real machine operations. The SPS beam test observed that the SEY was reduced to 1.5 after 4 days' scrubbing [13]. The estimated electron 
dose in first 24 hours is about $0.5 \mathrm{mC} / \mathrm{mm}^{2}$. The beam scrubbing effect in later 3 days is believed less than the first day, due to the reduced dose. More effective beam scrubbing within more acceptable period of time would require beams with higher intensity, which carries high risk of the machine damage.

The lowest SEY of TiN coating is about $1.5[14,15]$, where the coating quality control is a critical issue. The application results of TiN coating at PSR is mixed [16].

The bench test results of NEG coating at SLAC are in agreement in general. For example, after 2 hours of activation with $210^{\circ} \mathrm{C}$, peak SEY of both TiZrV coated on steel with $2 \mu m$ thickness and TiZrV coated on Al with $1 \mu \mathrm{m}$ thickness had reduced to 1.2. Moreover, after 40 days of exposure to a vacuum of $5 \times 10^{-10}$ Torr, the SEY was stabilized at 1.4 [15].

\subsection{Electron desorption rate}

In a measurement using $500 \mathrm{eV}$ electrons as projectile, the gas desorption rate of a NEG coated surface is compared with the stainless steel surface baked at $300^{\circ} \mathrm{C}$ in two hours [17]. Desorption rate of $\mathrm{H}_{2}$ is $10^{-2}$ for steel, and $2 \times 10^{-4}$ for activated NEG coating. Desorption rate of CO is $8 \times 10^{-3}$ for steel, and $10^{-4}$ for activated NEG coating. In general, the electron desorption rate is reduced by factors of 10 to 100 for NEG coating.

Comparing with the SEY, the information on electron desorption rate of NEG is limited, especially of the saturated NEG.

\subsection{Ion desorption rate}

The desorption rates of steel and NEG coating have been compared in several measurements for either normal or grazing incidents.

The ion desorption experiments of NEG coating compared with the steel have been carried out at GSI with normal incident angle [18], and at CERN LINAC3 with 89.2 degree grazing angles [19]. The results are mixed.

At RHIC, beam ions were steered to stainless steel beam pipes and the nearby NEG coated pipe surface to observe the ion desorption effects [20]. The incident angles were approximately $1 \mathrm{mrad}$, and the desorbed gases were measured by the vacuum gauges. The calibration of the desorption could not be accurately determined, since the knowledge of the NEG pumping was not sufficient. However, the steel and NEG surface in the experiment are close 
enough that the desorption rates of the NEG coating and steel could be compared. The result was that the NEG coating desorption rate is lower than the baked steel surface by a factor of 2 to 3 .

In the experiment at BNL Tandem Van de Graaff [4], a NEG coated pipe containing a steel liner is used as a target of the ion bombardment at shallow angles. The NEG pumping factor is not excluded, but it functions equally for the steel and NEG surface bombardment. The result is that the NEG coating desorption rate is about $30 \%$ of the steel. By determining the pumping speed including the ion pumps and NEG coating, the desorption rate could be calculated.

\subsection{Ion scraping effects}

For the effects of the ion scraping on the chamber wall, it is of special interest to determine the desorption rate at the very shallow incident angles. There are two reasons [21].

1. The incident angle associated with the beam halo scraping is much smaller than $1 \mathrm{mrad}$. The beams with $1 \mathrm{mrad}$ incident angle interate with the beam pipes more like penetrating rather than scraping [22].

2. It is very difficult to determine the desorption rate under these conditions.

In [23], it has shown that for ions with the energy of $1 \mathrm{MeV} / \mathrm{u}$ or higher, the secondary electron yield follows the line of $1 / \sin \theta$ up to about $0.6 \mathrm{mrad}$, where $\theta$ is the incident angle. Instead of to collect secondary electrons, the bias voltage at the target was measured to determine the SEY.

The measurement of the ion desorption rate with shallow incident angles is more difficult.

With incident angles of $75 \mathrm{mrad}$ and $12 \mathrm{mrad}$, the desorption rate of $\mathrm{Pb}^{53+}$ with 4.2 MeV/u on steel and NEG surfaces were measured [24]. The shallower angle of $12 \mathrm{mrad}$ yields higher desorption with a factor of approximately 2 .

The desorption rate of $K^{1+}$ with $25 \mathrm{keV} / \mathrm{u}$ on steel target with incident angles from $150 \mathrm{mrad}$ to $30 \mathrm{mrad}$ was reported in [25]. The desorption rate increases when the incident angles become shallower. However, it was peaked at $50 \mathrm{mrad}$, and after that it is reduced. The low energy of potassium ions used in the experiment may be a factor for this less than $1 / \sin \theta$ in the scraping effect. 
Using $1 \mathrm{MeV} / \mathrm{u} A u^{15+}$ with incident angles from $100 \mathrm{mrad}$ to $10 \mathrm{mrad}$, the desorption rate of both steel and NEG (activated at $300^{\circ} \mathrm{C}$ ) have shown clear relation of $1 / \sin \theta[4]$. This is shown in Fig.3. Since the pumping of the chamber is not calibrated, the ion impact induced pressure rise versus the beam intensity is used to measure the desorption rate.

\section{Discussions}

In this section, we discuss several related aspects on the application of saturated NEG coatings.

\subsection{Mechanism of NEG surface properties}

The key to improve the pumping capability of NEG coating is to grow very rough film, and to make the surface capacity to reach that of bulk getters [1]. The roughness is suspected to be the most important mechanism of the NEG surface properties for at least the reduced SEY [26]. The evidences supporting this argument include:

1. The improvement of SEY by TiN coating depends on the coating conditions. Investigations reveal that the favorable coating conditions actually yield rougher surface [14]. This shows that it is the surface roughness rather than the material that is important in improving SEY.

2. Significant improvement of SEY on grooved surface shows further the importance of geometrical surface properties [27]. Simulations reveal that with the grooves, the secondary electrons have larger chance to hit the wall and get absorbed. The deeper the groove, the smaller the SEY.

Some attempts to investigate the surface properties can be found in [22]. It is, however, less clear for other relevant NEG surface properties, i.e. electron desorption rate, ion desorption rate, and ion scraping effects.

\subsection{Why NEG coatings?}

It is of interest to compare NEG coatings with other possible chamber surface improvements for the experimental background reductions. 


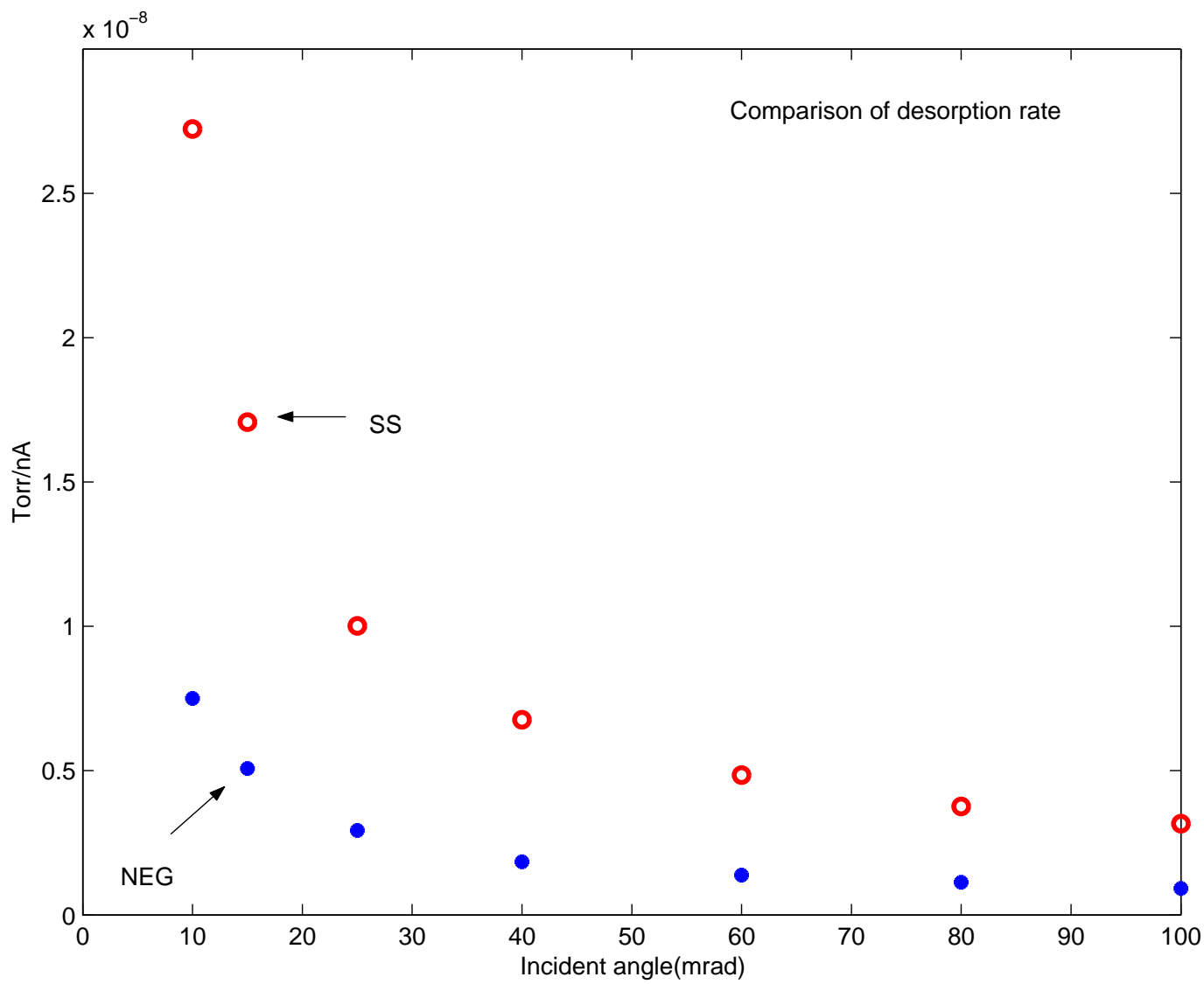

Figure 3: Desorption rates for $1 \mathrm{MeV} / \mathrm{u} A u^{15+}$ ions bombardment on steel and NEG surfaces. The NEG coating is activated at $300^{\circ} \mathrm{C}$, and steel target is a liner installed in the NEG pipe. The ion impact induced pressure rise versus the beam intensity is used to measure the desorption rate. 
1. Beam scrubbing for reducing SEY. To achieve comparable SEY with the saturated NEG coatings, very large electron dose is required. Both the machine time and the high risk in possible machine damage are of concern.

2. Beam scrubbing for reducing ion desorption. The beam scrubbing to reduce the ion desorptions [19] is much more difficult than the beam scrubbing in reducing SEY, since that only the part of the chamber directly hit by the beam ions has a chance to improve. The problems with beam scrubbing to reduce SEY also apply to the beam scrubbing to reduce ion desorptions.

3. TiN coating. Further improvements are needed to catch up with the NEG coatings in the SEY. Other properties relevant to experimental background are little known for the TiN coating.

4. Grooved surface. The SEY reduction has been reported [28], but other properties are not clear yet.

NEG coatings with the known properties that experimental background reduction needed, therefore, are in a better position to be further investigated, developed, and applied in this front.

\subsection{Further development}

In addition to the questions discussed above, there are also possible further development in saturated NEG coatings for the applications at interaction regions. These issues include:

1. Coating improvement

- Substrate material.

- Coating composition.

- Thickness.

- Coating conditions.

- Pd overlayer, pros and cons.

2. Saturatings 
- RHIC has tested nitrogen, which is not a complete coverage.

- Others include to use water, carbon monoxide, and carbon dioxide.

- Conditions and the subsequent consequences in saturating.

3. Applications

- Aging and lifetime.

- Possible damage and evaluation.

- Installation and maintenance.

\section{Acknoledgment}

I would like to thank C. Benvenuti, H.C. Hseuh, and P. Manini for helpful discussions.

\section{References}

[1] C. Benvenuti, Proc. PAC01, p.602, Chicago, 2001.

[2] P. Chiggiato and R. Kersevan, Vacuum 60, p. 67, 2001.

[3] B. Henrist, N. Hilleret, C. Scheuerlein, M. Taborelli, Applied surface science, 172, pp. 95-102, 2001.

[4] P. Thieberger, et al, http://www.cadops.bnl.gov/AP/PressureRise/50811PeterT.pdf, 2005.

[5] S.Y. Zhang, C-A/AP/107, BNL, 2003

[6] W. Fischer et al, Proc. EPAC04, p.917, Lucerne, Switzerland, 2004.

[7] F. Pilat et al, Proc. PAC05, p.4281, Knoxville, TN, 2005.

[8] S.Y. Zhang and D. Trbojevic, Proc. PAC07, Albuquerque, NM, 2007.

[9] A. Rossi, Proc. ELOUD04, p.113, Napa, CA, 2004,

[10] S.Y. Zhang et al, Proc. PAC05, p.4308, Knoxville, TN, 2005. 
[11] S.Y. Zhang et al, Proc. EPAC06, Edinburgh, UK, 2006..

[12] N. Hilleret et al, Proc. PAC99, p2629, New York, NY, 1999.

[13] J.M. Jimenez, et al., Proc. PAC03, p.307, Portland, OR, 2003 .

[14] H.C. Hseuh, et al, Proc. ELOUD04, p.119, Napa, CA, 2004,

[15] F. Le Pimpec, R.E. Kirby, F. King, M. Pivi, Nucl. Instru. \& Methods in Phys. Res. A 551, pp. 187-199, 2005.

[16] R. Macek, Pressure rise workshop, BNL, Dec. 2003.

[17] P. Chiggiato, LHC-VAC/ChR/mpt, CERN, 2001.

[18] A. Kramer, Pressure rise workshop, BNL, Dec. 2003.

[19] E. Mahner, et al, Phys. Rev. ST Accel. Beams, 8, 053201, 2005.

[20] H. Huang, et al, Proc. EPAC 2006, Edinburgh, UK, 2006.

[21] S.Y. Zhang, Proc. HB-2004, p.216, Bensheim, Germany, 2004.

[22] P. Thieberger, et al, Phys. Rev. ST Accel. Beams, 7, 093201, 2004.

[23] P. Thieberger, et al, Phys. Rev. A61, 042901, 2000.

[24] E. Mahner, et al, Phys. Rev. ST Accel. Beams, 6, 013201, 2003.

[25] A.W. Molvik, et al, Proc. PAC05, p.194, Knoxville, TN, 2005.

[26] S.Y. Zhang and T. Roser, Proc. ELOUD04, p.5, Napa, CA, 2004.

[27] G. Stupakov and M. Pivi, Proc. ELOUD04, p.139, Napa, CA, 2004.

[28] M. Pivi, Proc. ELOUD07, Daegu, Korea, 2007. 